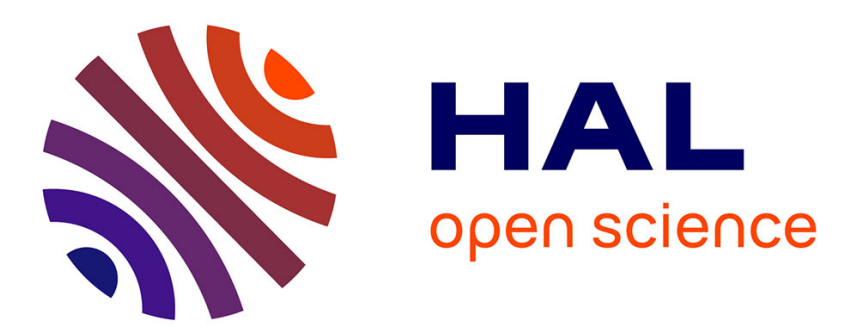

\title{
Stability of frozen water droplets on highly hydrophobic porous surfaces: Temperature effects
}

\author{
Stella M.M. Ramos, Christophe Pirat, Cécile Cottin-Bizonne
}

\section{To cite this version:}

Stella M.M. Ramos, Christophe Pirat, Cécile Cottin-Bizonne. Stability of frozen water droplets on highly hydrophobic porous surfaces: Temperature effects. Applied Surface Science, 2019, 469, pp.864869. 10.1016/j.apsusc.2018.11.027 . hal-02285884

\section{HAL Id: hal-02285884 \\ https://univ-lyon1.hal.science/hal-02285884}

Submitted on 6 Mar 2020

HAL is a multi-disciplinary open access archive for the deposit and dissemination of scientific research documents, whether they are published or not. The documents may come from teaching and research institutions in France or abroad, or from public or private research centers.
L'archive ouverte pluridisciplinaire HAL, est destinée au dépôt et à la diffusion de documents scientifiques de niveau recherche, publiés ou non, émanant des établissements d'enseignement et de recherche français ou étrangers, des laboratoires publics ou privés.

\section{(c)(1)}

Distributed under a Creative Commons Attribution| 4.0 International License 


\section{Accepted Manuscript}

Full Length Article

Stability of frozen water droplets on highly hydrophobic porous surfaces: temperature effects

Stella M.M. Ramos, Christophe Pirat, Cécile Cottin-Bizonne

PII: S0169-4332(18)33097-6

DOI: https://doi.org/10.1016/j.apsusc.2018.11.027

Reference: APSUSC 40872

To appear in: $\quad$ Applied Surface Science

Received Date: $\quad 11$ September 2018

Accepted Date: $\quad 4$ November 2018

Please cite this article as: S.M.M. Ramos, C. Pirat, C. Cottin-Bizonne, Stability of frozen water droplets on highly hydrophobic porous surfaces: temperature effects, Applied Surface Science (2018), doi: https://doi.org/10.1016/ j.apsusc.2018.11.027

This is a PDF file of an unedited manuscript that has been accepted for publication. As a service to our customers we are providing this early version of the manuscript. The manuscript will undergo copyediting, typesetting, and review of the resulting proof before it is published in its final form. Please note that during the production process errors may be discovered which could affect the content, and all legal disclaimers that apply to the journal pertain. 


\title{
Stability of frozen water droplets on highly hydrophobic porous surfaces: temperature effects
}

\author{
Stella M.M. Ramos*, Christophe Pirat, Cécile Cottin-Bizonne
}

Université de Lyon, Université Lyon 1 and CNRS, UMR5306, F-69622 Villeurbanne, France

\begin{abstract}
We report on an experimental study of water droplets freezing on highly hydrophobic surfaces decorated by a random distribution of nanometric pores. We particularly analyze the influence of the substrate temperature ranging between $23^{\circ} \mathrm{C}$ and $80^{\circ} \mathrm{C}$ on the freezing phenomenon and the temporal stability of the resulting frozen droplets. The cooling and the subsequent spontaneous freeze of droplets were obtained by quickly reducing the pressure inside the cell from $101 \mathrm{kPa}$ to $2 \mathrm{kPa}$. In all experiments the humidity rate was maintained very low (4\%), allowing us to minimize its influence on the freezing process. The experimental results show that: (i) the freezing process is initiated by homogeneous nucleation near the gas-liquid interface, and is delayed by a factor between 2 and 3 on the substrates at the highest temperature. (ii) three different mechanisms, influenced by the substrate's temperature, lead to the disappearance of the frozen droplets. Fragmentation and sublimation of frozen droplets are observed for all substrates' temperatures. A melting of the ice shell is observed for droplets on substrate at $80^{\circ} \mathrm{C}$. This temperature appears as a minimal value from which we can expect to better control the water freezing and the frost formation on heated hydrophobic surfaces.
\end{abstract}

Keywords: freezing, droplets, hydrophobic, frozen, melting

*Corresponding author: Stella M.M. Ramos

E-mail: stella.ramos-canut@univ-lyon1.fr.

Postal address: Institut Lumière Matière - Université LYON 1

Campus LyonTech - La Doua

F-69622 Villeurbanne Cedex France 


\section{Introduction}

Freezing of water droplets on solid surfaces has been studied with an increasing interest for both fundamental research [1-5] and a broad range of practical applications such as aircraft, wind turbines and marine structures [6-7]. Many of such applications are faced with the hazardous effect of ice accumulation in the mechanical device, which can seriously compromise the energy efficiency. It is thus not surprising that significant effort has been expended to develop surfaces that substantially reduce the ice adhesion or delay its formation [8-12]. Several approaches which prevent ice formation or reduce ice adhesion and accumulation have been proposed over the last decades. Some of these studies conclude that the ice adhesion reduces with increasing hydrophobicity of the surface, rendering the superhydrophobic surface a more interesting candidate for anti-icing applications. These surfaces, characterized by high water repellency and low friction at liquid-solid interface, are expected to minimize the ice or frost creation and their adhesion to the surface. A substantial amount of research has focused on the potential icephobic properties of such surfaces.

Thus, in the last few years, several authors [2, 13-14] have shown that a delayed ice accretion occurs on superhydrophobic surfaces. Others authors have correlated the contact angle hysteresis with the ice adhesion [15-16]. This statement and the role played by surface roughness on the icephobic behavior have been however critically questioned [17-21]. For example, Varanasi et al [17] reported that the frost formation inside the texture of superhydrophobic surfaces could compromise their effectiveness in reducing the ice adhesion, which increases with surface roughness. Jung et al [19] have shown that hydrophilic surfaces with nanometer $(\mathrm{nm})$-scale roughness and higher wettability present longer freezing delays compared with typical superhydrophobic surfaces with larger hierarchical roughness and very low wettability. In fact, the efficiency of anti-icing material can also be limited by the presence of frost on the surface whose formation is highly influenced by the environmental humidity [18,21-22]. On the other hand, more recently, the durability of the icephobic properties of superhydrophobic surfaces has been questioned [23-26] and some controversial conclusions appear.

These numerous investigations evidence clearly the influence of many parameters (e.g., hydrophobicity, surface roughness, environmental humidity and fragility of surface structures...) on the ice formation on a solid surface and its adhesion or stability. The role played separately by each one of them in the ice formation remains still unclear.

We report here an experimental study of water droplet freezing on highly hydrophobic surfaces. We particularly investigate the influence of the substrate temperature, ranging 
between $30^{\circ} \mathrm{C}$ and $80{ }^{\circ} \mathrm{C}$ in the freezing process and its temporal stability. In all experiments the humidity rate was maintained very low (4\%), allowing us to minimize its influence in the freezing process. Independently of the substrate temperature, the droplets freeze when the environing pressure inside the vacuum chamber is abruptly reduced. The freezing process is delayed at higher temperatures due to the increase of the heat transfer rate from liquid-solid contact interface. We observe that the thin ice layer formed melts in few seconds. We discuss the morphology adopted by the frozen droplet and its temporal evolution as a function of the substrate's temperature.

\section{Experimental section}

$55 \mu$ m-thick anodisc aluminum oxide membranes purchased from Sigma-Aldrich were used as substrate. The surfaces are patterned with a random distribution of nanometric pores characterized by a mean diameter of $200 \mathrm{~nm}$ and a mean center-to-center distance of $316 \mathrm{~nm}$ (see figure 1). These geometrical characteristics lead to a solid fraction in contact with liquid of $\sim 0.69$.

To provide stable highly hydrophobic surfaces these membranes were grafted with fluoroalkyl-silane $\left(\mathrm{C}_{16} \mathrm{~F}_{17} \mathrm{H}_{19} \mathrm{O}_{3} \mathrm{Si}\right.$, named FAS-17) molecules. For this purpose, the previously cleaned samples were exposed to FAS-17 vapor at low pressure for about $10 \mathrm{~h}$. To remove all excess reactants, these substrates were rinsed in two successive ethanol baths, dried in $\mathrm{N}_{2}$ flux and subsequently kept in a closed box for about $12 \mathrm{~h}$ prior to further manipulation.

Contact-angle measurements of ultrapure water drops (of volume $\sim 2 \mu \mathrm{L}$ ) were carried out through the standard sessile drop method. For such experiments, a homemade device was used, allowing the substrate temperature and the pressure inside the cell to be controlled independently. The pressure control is assured using a vacuum pump. For each experiment, a drop was gently deposited from a needle connected to a syringe pump on the surface enclosed in a glass chamber. Side-view images of the drops were recorded with a CCD camera for subsequent contact angle and contact radius measurements. The dynamic contact angle hysteresis $(\Delta \theta)$, defined as the difference between advancing $\theta_{\mathrm{A}}$ and receding $\theta_{\mathrm{R}}$ contact angles, was also determined from our experiments. For such measurements the needle of a syringe was kept inside the drop. The advancing contact angle is reached when the contact line moves consecutively to addition of liquid, while the opposite configuration (liquid removed from the drop) allows to measure the receding contact angle $\theta_{R}$. All measurements 
dealing with wetting characterization were performed under ambient conditions (temperature: $23 \pm 1{ }^{\circ} \mathrm{C}$ and relative humidity: $45 \pm 2 \%$ ). At least five different measurements were performed on different areas foreach sample.

To study the dynamics of water droplet freezing on heated substrates the same experimental device was used. In this case a small drop (of volume $\sim 3 \mu \mathrm{L}$ ), initially at room temperature, was gently deposited on the surface enclosed in a glass chamber. Then the pressure inside the chamber was reduced in few seconds $(<10 \mathrm{~s})$, from $101 \mathrm{kPa}$ to $2 \mathrm{kPa}$. During this process the relative humidity inside the box drastically decreases and stabilizes at $4 \pm 1 \%$. This low value allows us to conduct the present study in what we call the "dry" humidity condition. It is a relevant feature considering the well-established influence of the environing humidity in the nucleation and freezing process of a water droplet [18,21-22]. The temperature of the substrates was varied from $23^{\circ} \mathrm{C}$ to $80^{\circ} \mathrm{C}$. More than sixty different experiments were performed on samples at different temperatures. In all cases, the freezing of the water droplet occurs under low pressure $\left(\mathrm{P}_{\mathrm{s}}=2 \mathrm{kPa}\right)$ and low humidity rate $(4 \%)$. Note that this low pressure inside the chamber allowed us to control the humidity rate. Consequently, the only variable parameter in our experiments is the substrate temperature. Pictures were taken either with a CCD camera or with a high-speed camera (Phantom v5.1) operating with a scan rate of $6000 \mathrm{fps}$.

\section{Results and discussion}

\subsection{Wetting properties}

The advancing and receding contact angles measured on processed surfaces, at room temperature, were $\theta_{\mathrm{A}}=143^{\circ} \pm 3^{\circ}$ and $\theta_{\mathrm{R}}=118^{\circ} \pm 4^{\circ}$. These values result from a statistical study having taken into account measurements carried out on at least twenty samples. In our experiments, we used water drops having a static contact angle $\theta$ of $\sim 135^{\circ}$. The relative high contact angle hysteresis $\left(\Delta \theta=25^{\circ}\right)$ suggests that a partial pore's imbibition should occur. Thus, the water droplets should be found in an intermediate state between Cassie-Baxter [27] (in a fakir configuration) and Wenzel states [28]. The fakir configuration usually describes the apparent contact angle in the case where the liquid does not penetrate inside the solid asperities at all, so the contact is mixed: solid-liquid and liquid-gas. Besides the Wenzel approach is appropriate to model the case where the liquid completely fills the pores. A combination of these two approaches appears as the most realistic to describe the wetting properties of our surfaces. The presence of some irregularities in the porous distribution (like 
percolation between two or more rarely three pores, see fig 1) supports also the description proposed. Consequently, despite the high value of the static contact angle $\left(\theta \sim 135^{\circ}\right)$, our surfaces cannot be considered as superhydrophobic surfaces (usually characterized by a low $\Delta \theta$ value), but only as highly hydrophobic surfaces.

It is worth noticing that previous studies were conducted mainly on superhydrophobic surfaces [1-2,4-5,17-19]. Studies on structured hydrophobic surfaces are still missing, though such surfaces would be closer to potential applications.

\subsection{Freezing drops}

Freezing drops on substrates heated at $23^{\circ} \mathrm{C}$

Figure 2 shows a typical high speed image sequence capturing the different freezing steps of a droplet on a hydrophobic membrane and its interesting particularities. Two sequential stages can be distinguished. The first one deals with the nucleation and freezing process of a supercooled droplet that, in our experiments, starts with a spontaneous homogeneous nucleation at the gas-liquid interface (fig 2a-d). In these figures, the origin and direction of arrows mark the instantaneous location and direction of the freezing front, which moves continuously through the whole drop. The propagation of this front is very fast and within $\sim 14$ ms the droplet exhibits a cloudy appearance. This solidification time is in good agreement with the values measured in previous studies dealing with freezing of drops on cooled substrates $[1,12]$. We then observe ice nucleation with the formation of an ice shell. We now consider the stability and the temporal evolution of such droplet, which can present at least three different behaviors:

(i) The first one is seen in figs 2e-i. After total freezing the drop morphology changes, the drop moves and the contact between the drop and the solid substrate evolves. In about 1-2 seconds after nucleation, the ice shell breaks and shortly after the entire droplet is completely fragmented.

(ii) The second behavior is characterized by the presence of a liquid core inside the ice shell, as clearly evidenced in figure 3 . In this case, immediately after the freezing process, a thin ice feature (like a needle) is initially pushed out from the droplet. When it stops growing the ice shell is detached from the liquid drop and pushed up by the water drop that emerges inducing the formation of a water meniscus between the ice shell and the solid surface. The ice shell is thus gradually expelled, releasing the liquid part, that shows now a static contact angle of $\sim 60^{\circ}$. This value 
indicates a significant imbibition of the membrane's nanopores. Considering the initial hydrophobic characteristics of this surface, a water penetration will occur only above an intrusion pressure which can be estimated by $P_{\text {int }} \sim 2 \gamma \cos \theta_{0} / d$, where $\gamma$ is the surface tension of water, $\theta_{0}$ is the contact angle on the flat surface with the same functionalization and $\mathrm{d}$ is the pore diameter. By using the experimental data $\gamma \approx 75 \mathrm{mN} / \mathrm{m}$, surface tension of water at $0^{\circ} \mathrm{C}, \theta_{0}=115^{\circ}$ [29-30] and $\mathrm{d}=200 \mathrm{~nm}$, we found $P_{i n t} \sim 3 \mathrm{MPa}$. Evidently, according to this criterion, the pore's imbibition cannot naturally occur. This phenomenon can be expected only on droplets faced with the relatively high pressure.

In cases (i) and (ii) illustrated above (figs 2 and 3), the temporal evolution of the frozen drop leads to a release of the liquid core from the ice shell. Such an effect can be attributed to a strong increase of the pressure inside the droplet resulting from the expansion of the freezing water against the ice shell. The quasi-incompressibility of the water implies that a small fraction of the liquid droplet frozen is enough to induce the strong pressure increase. Previous studies, dealing with freezing drop on superhydrophobic surfaces, showed that the pressure inside the frozen drop can reach values of $\sim 2-4 \mathrm{MPa}$ [5]. Such pressures are high enough to induce, via a radial distribution of the internal pressure, an imbibition of the membrane's pores and consequently a wetting transition from hydrophobic to hydrophilic state.

(iii) A third particular behavior (found in about $20 \%$ of our experiments) for the frozen drop is evidenced, and displayed in Figure 4a. In this case, just after the freezing the drop adopts a completely spherical shape, it moves and rolls freely over the surface indicating a very low adhesion between the frozen drop and the solid substrate. Two effects can contribute to this behavior. In such configuration, the shape of the drop remains stable all the time, while its surface adopts a rough appearance (like a "tennis ball") which increases with the time. This induces a reduction of the droplet surface contact area and may promote the droplet mobility. On the other hand, as mentioned above, the behavior of the droplets (before and after freezing) and their rolling ability on solid surfaces are strongly influenced by the ambient humidity [18,21-22]. The particular "dry" humidity conditions used in our experiments may also contribute to increase the drop mobility. In fact, it reduces the probability that the surface might be contaminated by small ice crystals that could act as nuclei inducing a non-controlled drop freezing or a frost 
formation occuring on surfaces. The occurrence of these events evidently should affect the droplet displacement. The evolution of both the contact radius and the contact angle of the droplet (before and after freezing) is displayed in Fig 4b. As can be seen, the experiment starts with liquid drop having a static contact angle of $135^{\circ}$. Few seconds later, when the drop is freezing, the contact angle instantaneously increases reaching values in the $160^{\circ}-170^{\circ}$ range. In the meantime, the contact radius jumps to lower values, and is drastically reduced. The droplet remains in such configuration during about $65 \%$ of its lifetime. This very low friction at liquid-solid interface, responsible for the high mobility of the drop, characterizes a so-called "Cassie ice state" [25], an attracting configuration in view of many applications in the icephobic field [6-7]. Additionally, it has to be noticed that the disappearance of the droplet in this case occurs by a relatively slow sublimation process. The slow evolution results mainly from the geometric characteristics of the droplet imposed by the much higher contact angles ( $>160^{\circ}$ ). In this case, where the contact between the droplet and the solid surface is limited to a very narrow region, the evolution of the droplet is mainly controlled by the drop-gas interface and by the evaporation process.

In summary, our experiments evidence two ways leading to frozen droplets disappearance on substrates at $23^{\circ} \mathrm{C}$, that we call "fragmentation/sublimation" (dealing with explosion/ejection of the ice shell) and "pure" sublimation (when no fragmentation of the frozen droplet is observed).

\section{Freezing drops on substrates heated at $80^{\circ} \mathrm{C}$}

Next we focused our investigation in the freezing process of water droplets on substrates heated at $80^{\circ} \mathrm{C}$. In this case, the first stage that consists in the freezing and nucleation processes when no of the droplet appears similar to the one observed previously (see film in Supporting Information) for droplets at $23^{\circ} \mathrm{C}$. A homogeneous freezing front moving from the top to the base of drop is observed. The average solidification time measured from our experiments is $\sim 41 \mathrm{~ms}$. Clearly the heat transferred from the solid-liquid interface to the liquid drop is not enough to avoid its fast freezing. The evaporation of the liquid is thus initially dominant. The propagation velocity of the freezing front is however slowed down. For drops of the same size the following relation was established between the two extrema velocities, $\mathrm{V}_{30^{\circ} \mathrm{C}} \approx 2.5 \times \mathrm{V}_{80^{\circ} \mathrm{C}}$. 
Dealing with the temporal evolution of the frozen droplet on the warm substrates, the situation is more complex. In this case, the liquid core of the frozen droplet undergoes two antagonistic effects: a heat transfer from the warm substrate and an endothermic sublimation process at the ice surface.

The temperature gradient created inside the droplet promotes a convective flow which should induce some instabilities. The behaviors more frequently adopted by the fronzen droplets are the following :

(i) The first one and the most representative, that we will call melting, is illustrated in Figure 5. After partial freezing, the morphology of the frozen drop is drastically modified versus time, as can be seen from Fig 5b-f, where the ice shell appears to consist of small structures more or less intricated. Such features probably evidence small convective cellules as those characteristics of the Benard-Reyleigh instabilities (see Supporting Information). These cells disappear with time and gradually the thin ice layer melts. A melting time of $6 \mathrm{~s}$ and a propagation velocity of the melting front of $\sim 0.35 \mathrm{~m} / \mathrm{s}$ was determined from Figs $5 \mathrm{e}-\mathrm{h}$. This process is about three orders of magnitude slower than the freezing process of the initial water droplet. Then in Fig. 5(g-j) a gradual melting of the ice shell wrapping the liquid drop is evidenced. The heat transferred from the substrate to the drop is thus dominating in time. In a last step, the restored liquid drop evaporates up to the end of its life. It is worth noticing that in the present case, the porous membrane holds its hydrophobic characteristics, a static contact angle of $116^{\circ}$ was measured. Consequently the effect of the substrate temperature on the freezing of a water drop is twofold: It delays by a factor between 2 and 3 the solidification time. Then, in few seconds, it melts the solid ice shell avoiding thus that a stable ice (or frost) layer settles down on the substrate. Such an effect presents obvious pratical interest.

(ii) The second most frequent temporal evolution of frozen drops consists in a defrosting of the ice shell followed by a sublimation effect (see images in Supporting Information). In this case, the ice shell is gradually replaced by multiples spindly frost crystals that cover all the drop surface. Initially small and dense, such structures become very thin and more sparse with time, and finally sublimate. The heat transfer from the warm substrate surface to the droplets contributes to accelerate here this process. 
(iii) A more reduced number of the frozen drops evolve towards an ejection or fragmentation of the ice shell. Affected by the substrate temperature, the thickness of the ice shell is probably here thinner than on other surfaces set at lower temperatures. In this case, the pressure inside the droplet is probably not high enough to produce effects like the explosion of the ice shell.

To complete our investigation on the influence of the substrate temperature on water droplets submitted to a drastic pressure reduction, experiments were also carried out on surfaces heated at, $40^{\circ} \mathrm{C}$ and $60^{\circ} \mathrm{C}$. Table 1 summarizes, for all temperatures investigated, the occurrence of the different configurations adopted by droplets after freezing. Each of them can be associated to a specific physical mechanism (fragmentation, sublimation, melting/defrosting) responsible for the drop disappearance. From Table 1 three main features can be derived :

(i) The phenomenom of fragmentation and ejection of the ice shell is less frequently observed at highest temperatures. In this case, the increase of the substrate temperature should reduce the thickness of the ice shell and consequently reduce also the internal pressure in the encapsulated liquid droplet.

(ii) The dissapearence time of the droplet by sublimation increases with the temperatures in the range $23^{\circ} \mathrm{C}-60^{\circ} \mathrm{C}$. The substrate temperature providing an energy to the frozen droplet favorises the endothermic phase transtions as the sublimation.

(iii) There is a temperature threshold above which, even if not avoiding water freezing, liquid droplet can be restored by the ice melting. Combined with the dry humidity conditions of our experiments, it avoids the frost formation preserving the hydrophobic properties of the surfaces.

Finally, with these experiments we have identified three different ways (illustrated in Figure 6) leading to the disappearance of the frozen droplets and have characterized the influence of the substrate temperature.

Keeping in mind potential applications, it is interesting to underline the determination, from the experimental data presented above, of a temperature value imposed to the substrates that allows at least partially to control the frost and freezing process of water droplets on hydrophobic surfaces. This particular value of temperature can be considered as a minimal value from which we can expect to better control the water freezing and the frost formation on heated hydrophobic surfaces. 


\section{Conclusion}

We have experimentally investigated the freezing process of water droplets and its temporal stability on highly hydrophobic surfaces. In particular, we have highlighted the influence of the substrate's temperature. From our experiments, it clearly appears that independently of the substrate temperature, the droplets freeze when the environing pressure inside the vacuum chamber is abruptly reduced to $2 \mathrm{kPa}$. The freezing process is delayed at higher temperatures due to the increase of the heat transfer rate from liquid-solid contact interface. Our main findings are twofold: (i) The stability of the frozen droplets and its subsequent disappearance is controlled by three different mechanisms (fragmentation, sublimation and melting), influenced by the substrate's temperature. Associated or separately, fragmentation and sublimation of frozen droplets are systematically observed. In the case of a "pure" sublimation (in the absence of a droplet fragmentation) a "Cassie ice state" can be reached. The very low friction at liquid-solid interface characterizing such configuration improves the robustness of these hydrophobic surfaces for icephobicity. A melting of the ice shell is observed for droplets on substrate at $80^{\circ} \mathrm{C}$. This temperature appears as a minimal value above which the liquid droplet can be restored by the ice melting. Combined with the "dry" humidity conditions it avoids the frost formation preserving thus the hydrophobic properties of the surfaces. (ii) The thermal energy brought by the substrate heating leads to instabilities and convective cells are observed. Two timescales associated to the freezing and melting processes are identified (freezing time $\sim$ ms and melting time $\sim \mathrm{s}$ ) on heated substrates.

Finally, this experimental study deepens our understanding of the influence of the substrate's temperature on the freezing of water droplets and the mechanisms leading to the frozen droplet disappearence. A better control of these two processes on heated hydrophobic surfaces can be thus expected. 


\section{References}

[1] S. Jung, M.K. Tiwari, N.V. Doan, D. Poulikakos, Mechanisms of supercooled dropled on surfaces, Nature communications, 3 (2012)1-8, 2012.

[2] P. Tourkine, M. Le Merrer and D. Quéré, Delayed freezing on water repellent materials, Langmuir 25 (2009) 7214-7216.

[3] A. G. Marin, O.R. Enriquez, P. Brunet, P. Colinet and H. Snoeijer, Universality of tip singularity formation in freezing water drops, Phys. Rev. Lett. 113 (2014) 054301-5.

[4] T.M. Schutzius, S. Jung, T. Maitre, G. Graeber, M. Kôhme and D. Poulikakos, Spontaneaous droplet trampolining on rigid superhydrophobic surfaces, Nature 527 (2016) 82-85.

[5] S. Wildeman, S. Sterl, C. Sun and D. Lohse, Fast dynamics of water droplets freezing from the outside-in; Phys. Rev. Lett. 118 (2017) 084101-1-4.

[6] V. F. Petrenko and R.W. Whitworth, Physics of ice (Oxford University Press, New York, 1999).

[7] J. L. Laforte, M. A. Allaire, and J. Laflamme, State-of-the-art on power line de-icing, Atmos. Res. 46, (1998) 143-158.

[8] E. Moallem, T. Hong, L. Cremaschi and D. E. Fisher, Effects of surface coating and water retention on frost formation in microchannel evaporators (ASHRAE RP-1589), HVAC\&R, 19 (2013) 347-362.

[9] V. F. Petrenko and S.Peng, Reduction of ice adhesion to metal by using self-assembling monolayers (SAMs), Can. J. Phys. 81 (2003) 387-393.

[10] A. J. Meuler, J. D. Smith, K. K. Varanasi, J. M. Mabry, G. H. McKinley, and R. E. Cohen, Relationships between water wettability and ice adhesion, ACS Appl. Mater. Interfaces, 2 (2010) 3100-3110.

[11] M. Zou, S.Beckford, R. Wei, C. Ellis, G. Hattton and M.A. Miller, Effects of surface roughness and energy on ice adhesion strength, Appl. Surf. Sci. 257 (2011) 3786-3792.

[12] P. Hao, C. Lv, X. Zhang, Freezing of sessile droplets on surfaces with various roughness and wettability; Appl. Phys. Lett. 104 (2014) 161609-1-5.

[13] M. He, J.X. Wang, H. L.Li, X.L. Jin, J.J. Wang, B.Q. Liu, Y.L. Song, Y.L. Song, Superhydrophobic film retard frost formation, Soft Matter 6 (2010) 2396-2399.

[14] D.P. Singh and J.P. Singh, Delayed freezing of water droplet on silver nanocolumnar thin film, Appl. Phys. Lett. 102 (2013) 243112-1-4.

[15] S.A. Kulinich and M. Farzaneh, How wetting hysteresis Influences ice adhesion strength on superhydrophobic surfaces? Langmuir 25 (2009) 8854-8856.

[16] A. Dotan, H. Dodiuk, C. Laforte, S. Kenig, The relation between water wetting and ice adhesion, J.Adh. Sci. Technol 23, 2009, 1907-1915. 
[17] L.Cao, A.K. Jones, V.K. Sikka, J.Wu and. Gao, Anti-icing superhydrophobic Coating, Langmuir 25 (2009) 12444-12448.

[18] K.K Varanasi, T. Deng, J.D. Smith, M. Hsu and N. Bhate, Frost formation and ice adhesion on superhydrophobic surfaces, App. Phys. Lett. 97 (2010) 234102-1-4.

[19] Stefan Jung, Marko Dorrestijn, Dominik Raps, Arindam Das, Constantine M. Megaridis, and Dimos Poulikakos, Are superhydrophobic surfaces best for icephobicity? Langmuir 27 (2011) 3059-3066.

[20] S. Chavan, J. Carpenter, M. Nallapaneni, J.Y. Chen and N. Miljkovic, Bull water freezing dynamics on superhydrophobic surfaces, Appl.Phys. Lett. 110 (2017) 041604-1-5.

[21] S.A. Kulinich, S. Farhadi, K. Nose and X.V. Du, Superhydrophobic surfaces: Are they really ice-repellent?, Langmuir 27 (2011) 25-29.

[22] J.B. Boreyko, B.R. Srijanto, T.D. Nguyen, C. Vega, M. Fuentes-Cabrera and P.C. Collier, Dynamic defrosting on nanostructured superhydrophobic surfaces, Langmuir 29 (2013) 9516-9524.

[23] S.A. Kulinich and M. Farzaneh, Ice adhesion on super-hydrophobic surfaces, Appl. Surf. Sci. 255 (2009) 8153-8157.

[24] Y. Wang, J. Xue, Q. Wang, Q. Chen Q. and J. Ding, Verification of icephobic/anti-icing properties of a superhydrophobic surface, Appl. Materials and Interfaces 5 (2013) 3370 3381.

[25] H. Sojoudi, M. Wang, N.D. Boscher, G.H. McKinley and K.K. Gleason, Durable and scalable icephobic surfaces: similarities and distinctions from superhydrophobic surfaces, Soft Matter 12 (2016) 1938-1963.

[26] L. Oberli, D. Caruso, C. Hall, M. Fabretto, P.J. Murphy, D. Evans, Condensation and freezing of droplets on superhydrophobic surfaces 210 (2010) 47-57.

[27] D. Cassie, S. Baxter, Wettability of porous surfaces, Trans. Faraday Soc. 40 (1944) 546551.

[ 28] R.N. Wenzel, Resistance of solid surfaces to wetting by water, Ind. Eng. Chem. 28 (1936) 988-994.

[29] N. Saleema, D.K. Sarkar, R.W. Paynter, W.-G. Chen, Superhydrophobic aluminum alloy surfaces by a novel one-step process, Appl. Mater. Interfaces 2 (2010) 2500-25002.

[30] N. Saleema, D.K. Sarkar, D. Gallant, R.W. Paynter, X.-G. Chen, Chemical nature of superhydrophobic aluminum alloy surfaces produced via a one-step process using fluoroalkylsilane in a base medium, Appl. Mater. Interfaces 3 (2011) 4775-4781.

[31] J. W. Biddle, V. Holten, J. V. Sengers, and M. A. Anisimov, Thermal conductivity of supercooled water, Phys. Rev. E 87 (2013) 042302-1-6.

[32] G. S. Kell, Density, thermal expansivity, and compressibility of liquid water from $0^{\circ}$ to $150{ }^{\circ} \mathrm{C}$ : Correlations and tables for atmospheric pressure and saturation reviewed and 
expressed on 1968 temperature scale, Journal of Chemical \& Engineering Data, 20 (1975) 97-105.

[33] P. Manneville, Rayleigh-Bénard Convection: Thirty Years of Experimental, Theoretical, and Modeling Work. in: Mutabazi I., Wesfreid J.E., Guyon E. (Eds) Dynamics of SpatioTemporal Cellular Structures. Springer Tracts in Modern Physics, vol 207. Springer, New York 2006, NY. DOI: https://doi.org/10.1007/978-0-387-25111-0_3 
Table 1: Main configurations adopted by the frozen droplets.

\begin{tabular}{|c|c|c|c|}
\hline T $\left({ }^{\circ} \mathrm{C}\right)$ & \multicolumn{3}{|c|}{ Frequency observation of different phenomena (\%) } \\
\hline & Fragmentation & Sublimation & Melting \\
\hline 23 & 80 & 20 & - \\
\hline 40 & 72 & 28 & - \\
\hline 60 & 60 & 40 & 45 \\
\hline 80 & 25 & 30 & - \\
\hline
\end{tabular}


Figure 1: Scanning electron microscope image of the nanoporous aluminum oxide membrane. 
(a)

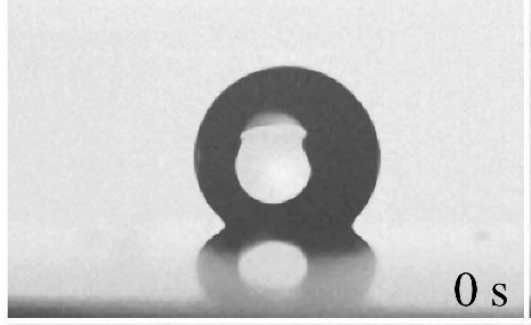

(d)

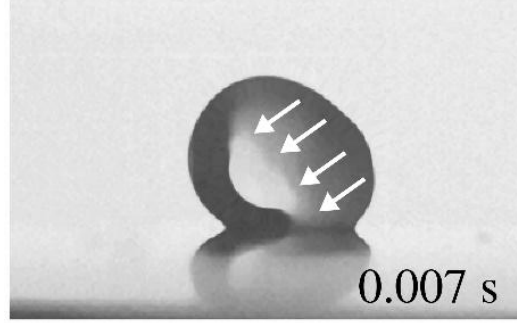

(g)

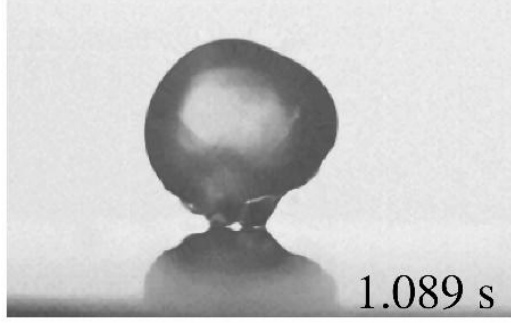

(b)

(e)

(c)
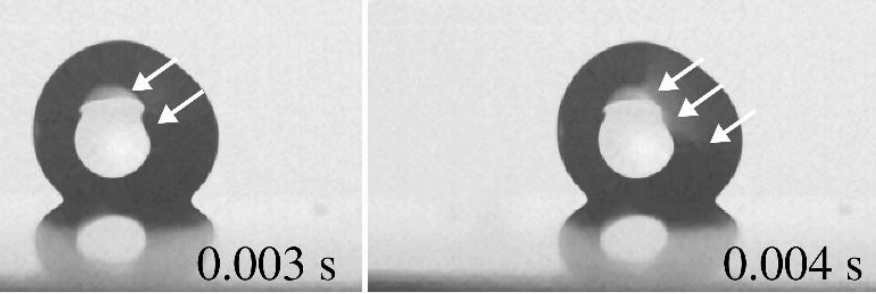

(f)

$0.796 \mathrm{~s}$

(h)
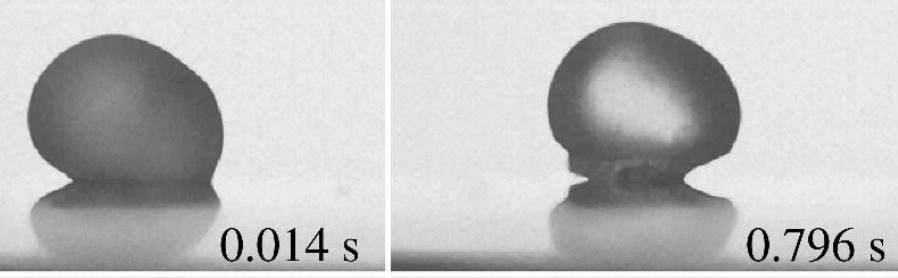

(i)
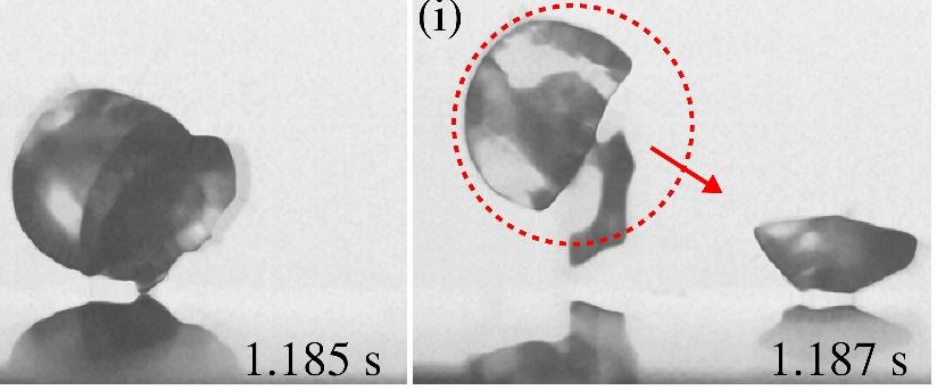

Figure 2: High speed recording of the freezing process of a water droplet on a substrate at $23^{\circ} \mathrm{C}$. The arrows indicate the instantaneous position and direction of motion of the freezing front. After few seconds the droplet cracks and finally explodes. 


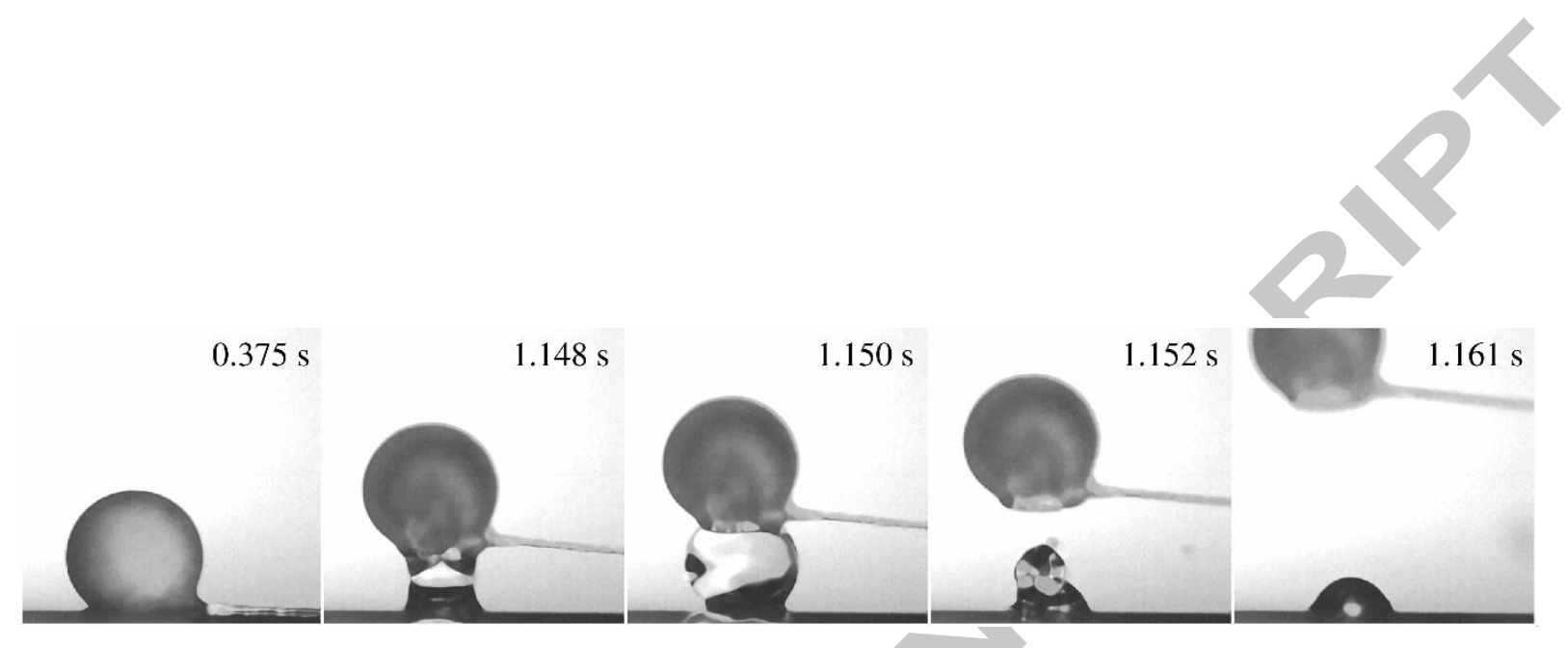

Figure 3: High speed recording the temporal evolution of the droplet after freezing. Evidences of a liquid core via ejection of the ice shell. 


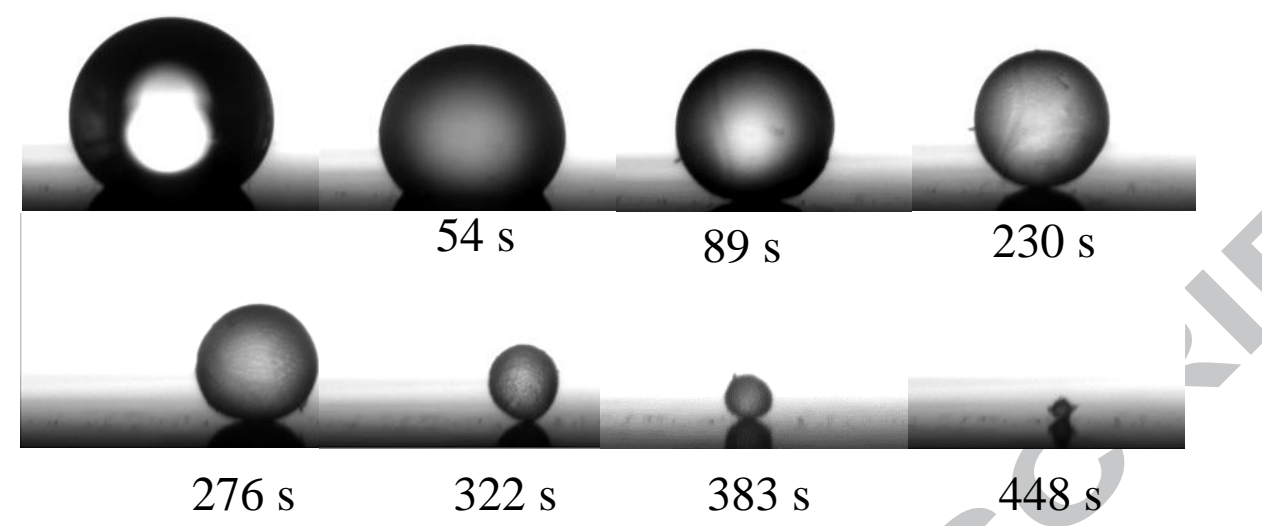

Figure 4a: Typical snapshots of freezing and sublimation of a water droplet on a surface at $23^{\circ} \mathrm{C}$.

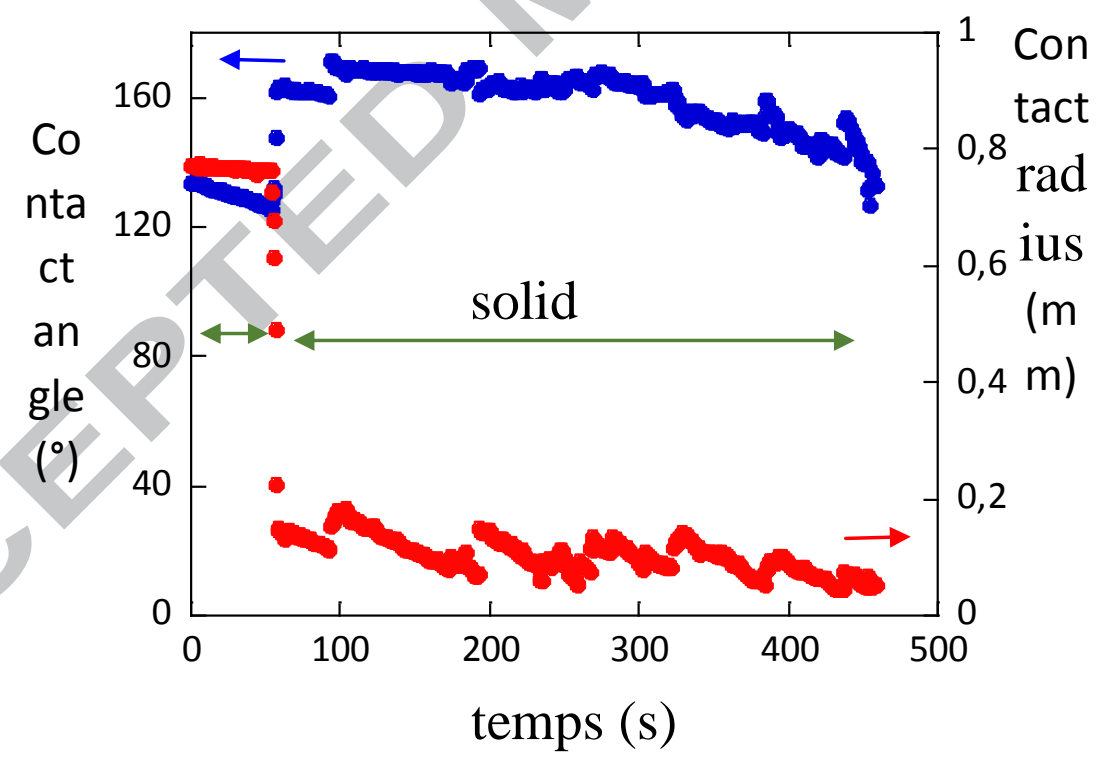

Figure 4b: Temporal evolution of the contact angle and the contact radius a water droplet before and after freezing. The partial solidification of the initial liquid droplet is indicated by the double arrows. 
(a)

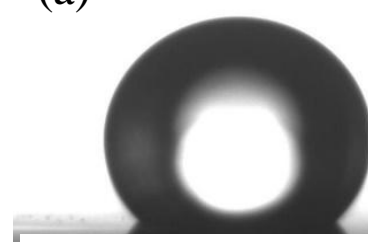

(d)

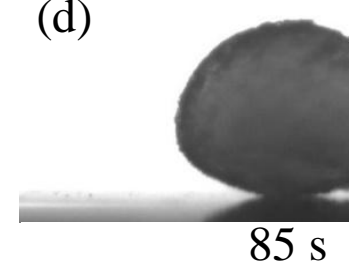

(g) (b)

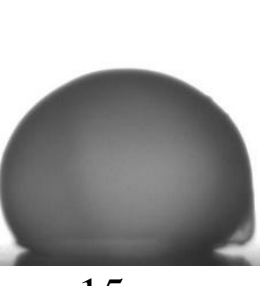

$15 \mathrm{~s}$

(e)

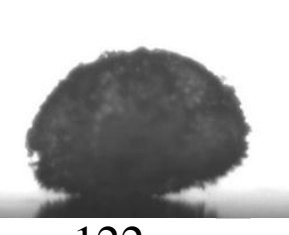

(c)

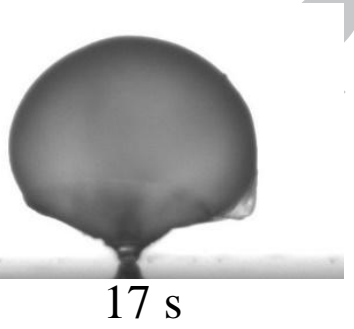

(f)

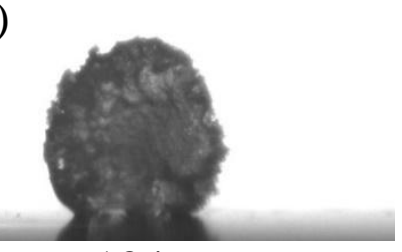

$134 \mathrm{~s}$

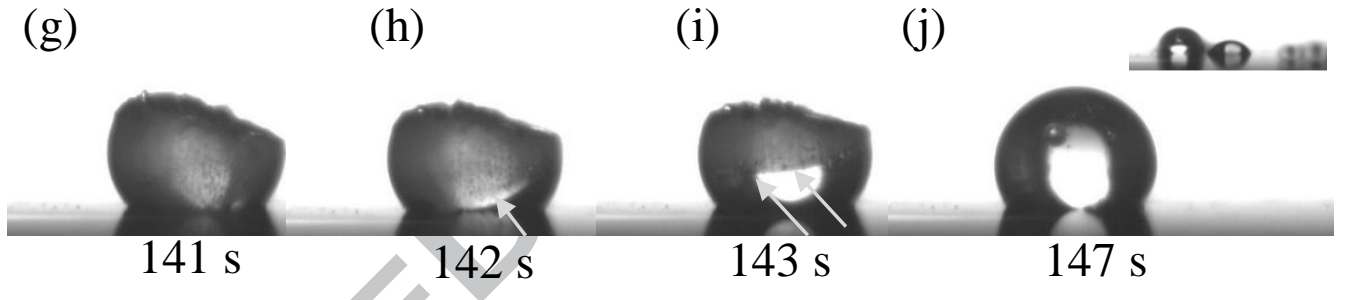

Figure 5: Freezing a water droplet on a substrate heated at $80^{\circ} \mathrm{C}$. Three phases are identified. droplet freezing $(\mathrm{a}-\mathrm{c})$; formation of small structures probably resulting from a competition between two fronts (heat and cold) inside the droplet (d-f); melting of the ice shell and evaporation of the released droplet $(g-j)$. 


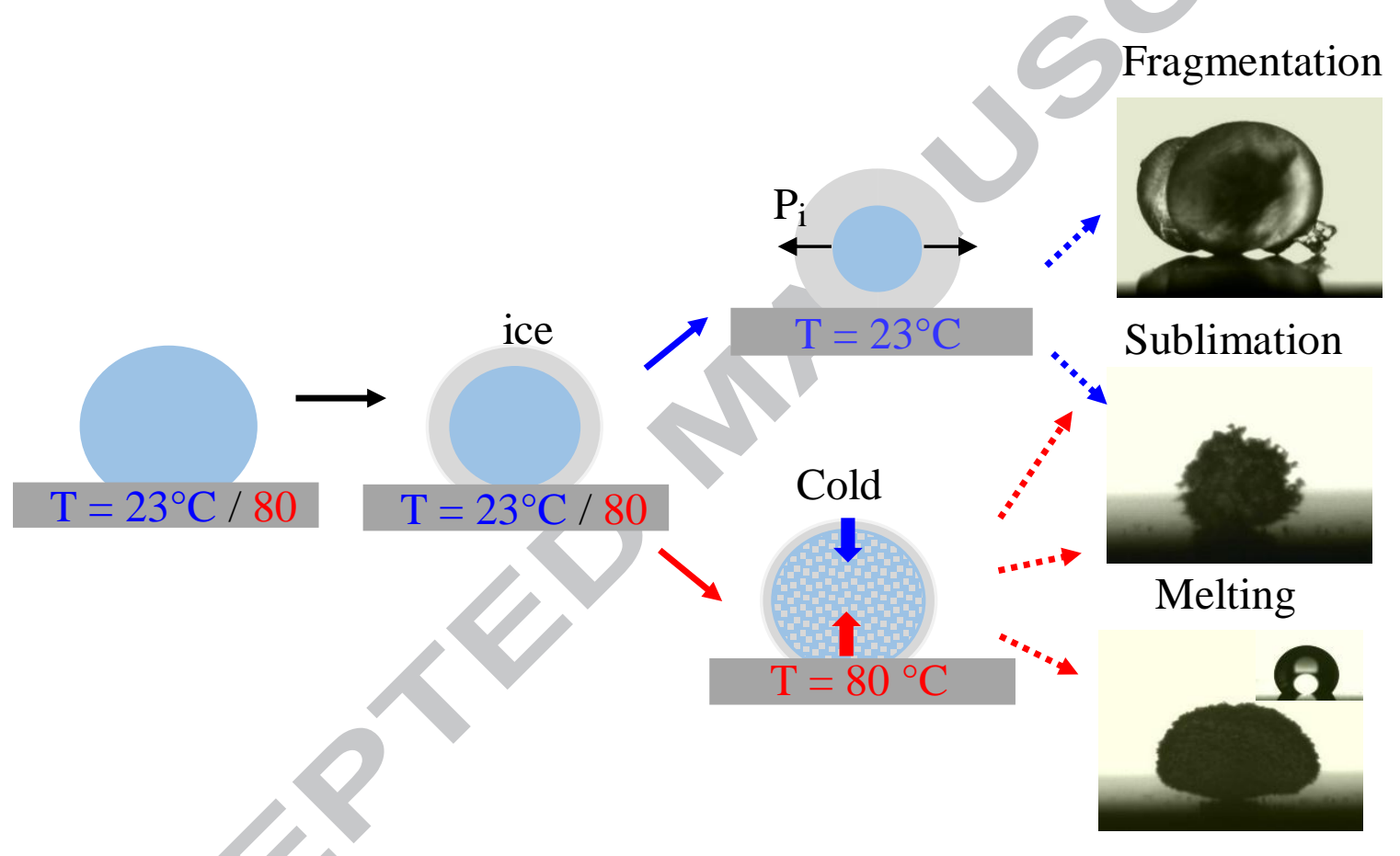

Figure 6: Summary of main results of this study. Placed on heated substrate the water droplets freeze. A liquid core is enveloped by an ice shell whose thickness should be influenced by the substrate temperature. The pressure inside the droplets, $\mathrm{P}_{\mathrm{i}}$, resulting from the quasi-incompressibility of the cooled water, leads to a drop fragmentation. $\mathrm{P}_{\mathrm{i}}$ is expected greater inside of thicker ice shell. At highest temperature, structures like "squirrel" resulting from a thermal gradient created inside the droplet, are initially observed. Then, the ice shell is finally melting. In some cases, a sublimation of such structures occurs. 


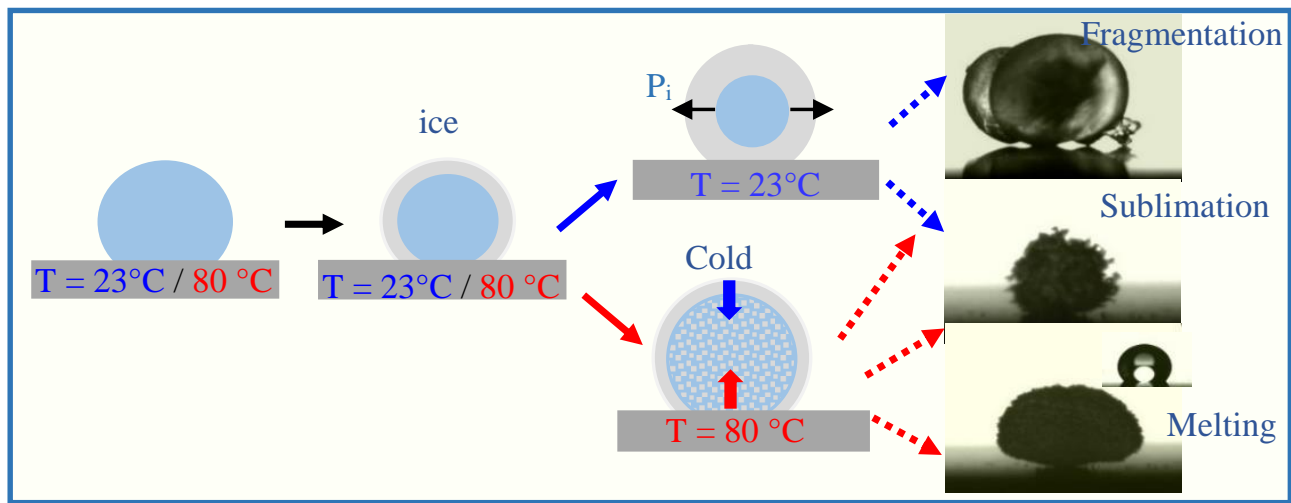




\section{Highlights}

- The freezing process of water droplets on highly hydrophobic surfaces was reported.

- Three different mechanisms, influenced by the substrate's temperature, control the frozen droplet disappearance.

- Thermal instabilities and convective cells are observed on heated substrates.

- A "Cassie ice state" can be reached on hydrophobic surfaces. 